\title{
O gengibre tem efeito sobre náuseas e vômitos em pacientes submetidos a quimioterapia?
}

\author{
Does ginger have an effect on nausea and vomiting in patients undergoing chemotherapy?
}

\author{
Adriele Silva Freitas ${ }^{a}$, Malaine Morais Alves Machadob ${ }^{b}$, Mônica Batista Soares ${ }^{a}$, \\ Marina Brito Campos ${ }^{c}$, Renata Costa Fernandes ${ }^{d}$ \\ a Nutricionista. Especialista em Hematologia e Hemoterapia pelo Hospital das Clínicas da Universidade Federal de Goiás (UFG).

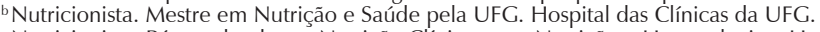 \\ c Nutricionista. Pós-graduada em Nutrição Clínica e em Nutrição e Hematologia e Hemoterapia. Hospital das Clínicas da UFG. \\ ${ }^{d}$ Nutricionista. Mestre em Nutrição e Saúde pela UFG. Hospital das Clínicas da UFG.
}

\begin{tabular}{l|l} 
RESUMO Introdução: O gengibre tem apresentado potencial efeito antiemético, porém ainda são escassos trabalhos que
\end{tabular} investigaram esse potencial em pacientes recebendo quimioterapia.

Objetivo: Avaliar o efeito do gengibre no controle de náuseas e vômitos em pacientes submetidos a quimioterapia.

Materiais e Métodos: Trata-se de um ensaio clínico, randomizado, triplo cego, controlado por placebo e cruzado. Foram recrutados 16 pacientes, dos quais 12 finalizaram a pesquisa. Os indivíduos foram divididos aleatoriamente para receber $1 \mathrm{~g}$ de extrato seco de gengibre ou placebo, quatro dias antes e após a quimioterapia. O estado nutricional foi avaliado pela Avaliação Subjetiva Global Produzida pelo próprio paciente. O instrumento para mensuração do desfecho principal foi o Multinational Association on Suportive Care in Cancer. O efeito do gengibre sobre o desfecho foi avaliado pelo teste de Wilcoxon Signed Rank para as variáveis ordinais e o teste de McNemar para as variáveis qualitativas.

Resultados: Entre o grupo gengibre e placebo não houve diferença quanto ao número de episódios de vômitos agudos e tardios, intensidade da náusea tardia, ocorrência de náusea aguda e tardia e ocorrência de vômitos agudos e tardios. Houve diferença na intensidade da náusea aguda, apresentando maior redução desta no grupo que recebeu o placebo.

Conclusão: A suplementação com $1 \mathrm{~g}$ de gengibre quatro dias antes e após da quimioterapia não apresentou efeito protetor em relação a náuseas e vômitos induzidos pela quimioterapia.

Palavras-chave: gengibre; náusea; vômito; neoplasias; quimioterapia.

Introduction: The ginger has a potential antiemetic effect, but there are few studies which investigate its effects on patients undergoing chemotherapy.

Objective: To evaluate the effect of ginger on the control of nausea and vomiting in patients undergoing chemotherapy.

Materials and Methods: This is a clinical, randomized, triple-blind, placebo-controlled, and crossover trial. Sixteen patients were recruited, of whom 12 completed the study. Subjects were randomly assigned to receive $1 \mathrm{~g}$ of a ginger dry extract or placebo four days before and after chemotherapy. The nutritional status was evaluated by the Global Subjective Evaluation Produced by the Patient. The instrument for measuring the main outcome was the Multinational Association on Suportive Care in Cancer. The effects were evaluated by the Wilcoxon test for the ordinal variables and by the McNemar test for the qualitative variables.

Results: When the ginger group was compared to the placebo group, there were no difference in the number of episodes of acute and late vomiting, the intensity of late nausea, the occurrence of acute and late nausea and the occurrence of acute and late vomiting. The groups differed in acute nausea, which were lower in the placebo group.

Conclusion: Supplementation with ginger is not effective to prevent nausea and vomiting induced by chemotherapy.

Keywords: ginger; nausea; vomiting; neoplasms; chemotherapy. 


\section{INTRODUÇÃO}

Os agentes antineoplásicos são amplamente utilizados no tratamento de neoplasias malignas. Por ser uma forma de tratamento sistêmico, a quimioterapia afeta indiscriminadamente todas as células do organismo, principalmente aquelas de rápida proliferação, produzindo efeitos adversos negativos, tais como mielossupressão, náuseas, vômitos, diarreia e alopecia ${ }^{1,2}$.

As náuseas e os vômitos estão entre os efeitos mais desagradáveis e frequentes da quimioterapia e afetam cerca de 70 a $80 \%$ dos pacientes que não realizam profilaxia antiemética. Entretanto, mesmo após profilaxia, 25 a 55\% dos pacientes submetidos à quimioterapia ainda apresentam vômitos e 50 a $75 \%$ apresentam náuseas, ocasionando implicações na qualidade de vida e adesão do paciente ao tratamento ${ }^{3}$.

A incidência das náuseas está relacionada com o potencial emético do quimioterápico utilizado, aliada à tolerância individual de cada paciente ${ }^{4}$. O potencial emético é expresso de acordo com a incidência de náuseas e vômitos associada à sua utilização. Um potencial emético menor que $10 \%$ é considerado mínimo, entre 10 a 30\% classifica-se como baixo, entre 30 a $90 \%$ moderado e valores acima de $90 \%$ encontram-se entre os agentes de alto potencial emético ${ }^{5,6}$.

Atualmente as plantas têm atraído considerável interesse no manejo de doenças. Entre as principais vantagens de sua utilização tem-se a menor ocorrência de efeitos colaterais e menor custo, quando comparados a drogas de origem sintética. O gengibre encontra-se entre as plantas eficazes no tratamento de náuseas e vômitos ${ }^{7}$. O gingerol, composto mais ativo do gengibre, tem atividade antagônica sobre o receptor colinérgico M3 e receptor serotoninérgico 5-HT3, apresentando bons resultados por acelerar o tempo de esvaziamento gástrico, evitando assim náuseas e vômitos ${ }^{8}$.

Apesar de já existirem estudos sobre efeitos positivos do uso do gengibre como antiemético, especialmente em gestantes, ainda são escassos trabalhos que investigaram esse potencial em pacientes recebendo quimioterapia, além de não haver consenso sobre dosagem, forma de apresentação e tempo de utilização $0^{9,7,10}$. Neste sentido, o objetivo do presente estudo foi avaliar o efeito do gengibre sobre náuseas e vômitos em pacientes submetidos a quimioterapia.

\section{MATERIAIS E MÉTODOS}

Trata-se de um ensaio clínico, randomizado, triplo cego, cruzado e controlado por placebo, realizado com pacientes em regime quimioterápico ambulatorial em um hospital público, no período de agosto a dezembro de 2017.
Foram elegíveis para a pesquisa pacientes oncológicos entre 20 a 80 anos, de ambos os sexos, com capacidade de engolir cápsulas, sendo tratados com quimioterapia (adjuvante, neoadjuvante, curativa, ou meios paliativos não exclusivos), submetidos a protocolos de quimioterapia (QT) de moderado a alto potencial emético; que tivessem sido submetidos a pelo menos um ciclo de QT com o mesmo agente quimioterápico e que tivessem sentido náuseas ou vômitos (em qualquer grau) como resultado desse tratamento. Foram excluídos pacientes com sensibilidade relatada ao gengibre ou que tivessem feito uso do mesmo na semana anterior ao início estudo; além daqueles em uso de anticoagulantes e/ou submetidos à radioterapia associada à $\mathrm{QT}^{9}$.

A coleta de dados foi realizada por nutricionistas treinadas previamente. Utilizou-se um questionário estruturado com questões sobre o perfil socioeconômico e demográfico, além de informações sobre as condições clínicas do paciente ${ }^{12}$. As entrevistas foram realizadas no próprio ambulatório de quimioterapia, em três momentos: antes da primeira intervenção $\left(\mathrm{T}^{\mathrm{A}}\right.$ e $\left.\mathrm{T} 0^{\mathrm{B}}\right)$, sendo aplicado a ambos os grupos o questionário sociodemográfico, instrumento de avaliação nutricional ASG-PPP e instrumento de náuseas e vômitos (MASCC $\left.{ }^{11}\right)$; e ao final da primeira $\left(\mathrm{T}^{\mathrm{A}}{ }^{\mathrm{A}}\right)$ e segunda intervenções $\left(\mathrm{T} 1^{\mathrm{B}}\right)$, foram aplicados novamente o instrumento de náuseas e vômitos, conforme Figura 1.

O diagnóstico nutricional foi determinado por meio da Avaliação Subjetiva Global Produzida pelo Próprio Paciente (ASG-PPP). Trata-se de uma ferramenta subjetiva, considerada padrão-ouro para avaliação nutricional de pacientes oncológicos, que os classifica em A - Bem nutrido, B - Desnutrição moderada ou suspeita de desnutrição e C - Gravemente desnutrido ${ }^{12}$.

$\mathrm{O}$ instrumento utilizado para quantificação das náuseas e vômitos foi o MASSC ${ }^{11}$ da Multinational Association on Suportive Care in Cancer, que considera náusea e vômitos agudos aqueles que acontecem em até $24 \mathrm{~h}$ após a quimioterapia, e náusea e vômitos tardios os que acontecem $24 \mathrm{~h}$ após da quimioterapia até 04 dias seguintes. A intensidade da náusea foi feita de acordo com escala numérica de um a 10 presente no instrumento utilizado, em que o participante respondia qual valor se assemelhava mais à intensidade da náusea que apresentou. A ocorrência tanto de náusea quanto dos vômitos foi classificada em sim ou não, também conforme instrumento.

Os participantes foram randomizados com o auxílio do site www.randomizer.org. Conforme Figura 1, foi incorporado um período de 15 dias entre os momentos (washout), com o objetivo de eliminar os possíveis efeitos da primeira fase. A avaliação do desfecho nos indivíduos foi feita 
em dois momentos: tempo inicial ou momento basal dos participantes que iniciaram a intervenção no grupo gengibre e momento basal daqueles que iniciaram no grupo placebo, e depois que cruzou-se a intervenção entre os grupos.

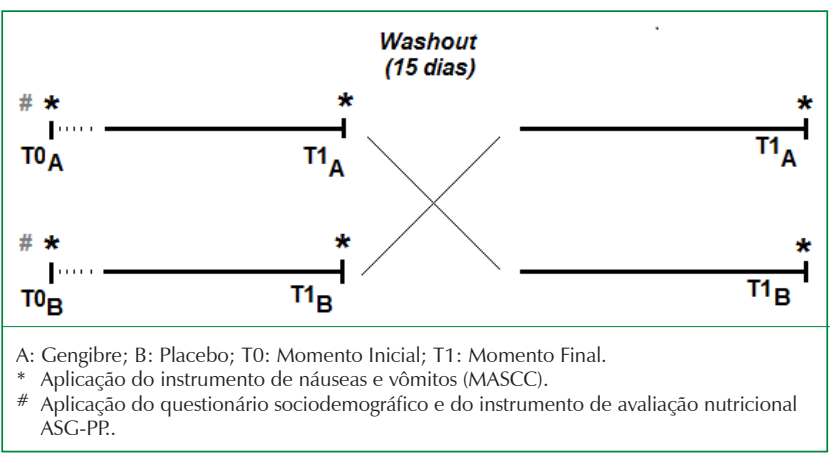

Figura 1. Protocolo experimental do estudo.

Todos os participantes receberam a intervenção com $1 \mathrm{~g}$ de extrato seco de gengibre ou $1 \mathrm{~g}$ de celulose microcristalina em momentos alternados, sendo duas cápsulas com $500 \mathrm{mg} /$ dia. O revestimento das cápsulas era escuro e opaco para que os participantes e os pesquisadores não pudessem identificar a intervenção recebida. Orientou-se ingerir as cápsulas pela manhã, quatro dias antes e quatro dias após a quimioterapia. Todos os participantes receberam medicamentos antieméticos, conforme protocolo implantado na instituição. Foi orientado aos voluntários que não ingerissem nenhum tipo de preparação contendo gengibre durante o período do estudo e que seguissem o mesmo padrão de ingestão alimentar e atividade física habituais.

Os dados foram digitados no Software Microsoft Excel (2010) com dupla entrada. A análise foi realizada no software $\mathrm{R}^{\circledR}$, sob supervisão de um estatístico. A normalidade das variáveis foi analisada utilizando-se o teste de Shapiro-Wilk.

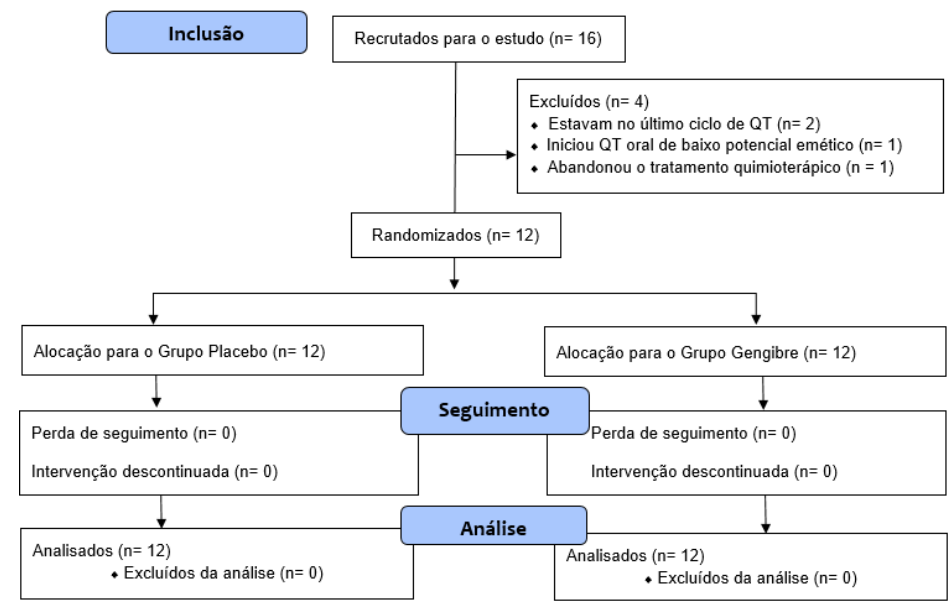

Figura 2. Fluxograma do estudo.
As variáveis foram descritas em frequências, média e desviopadrão, mediana e intervalo interquartil. $\mathrm{O}$ teste Exato de Fischer foi utilizado para avaliar a homogeneidade dos grupos no momento basal. Para a comparação do efeito do gengibre sobre as náuseas e vômitos associados à quimioterapia foi utilizado o teste de Wilcoxon Signed Rank para as variáveis ordinais e o teste de McNemar para as variáveis qualitativas. O nível de significância adotado foi de $5 \%(p<0,05)$.

O estudo foi aprovado pelo Comitê de Ética e Pesquisa do Hospital das Clínicas da Universidade Federal de Goiás, sob protocolo de número 2160599. Os indivíduos que aceitaram participar assinaram o Termo de Consentimento Livre e Esclarecido (TCLE). Esse estudo foi registrado no Registros Brasileiros de Ensaios clínicos (REBEC) com número RBR-33JPYY.

\section{RESULTADOS}

Foram recrutados 16 participantes, dos quais 12 finalizaram o estudo, conforme demonstrado na Figura 2. Os dados de caracterização da amostra encontram-se descritos na Tabela 1.

Tabela 1. Caracterização da amostra no momento inicial $(n=12)$.

\begin{tabular}{|c|c|}
\hline Variável & Valor \\
\hline $\begin{array}{l}\text { Sexo } \\
\text { Masculino } \\
\text { Feminino }\end{array}$ & $\begin{array}{l}4(44,44) \\
8(66,66)\end{array}$ \\
\hline Idade (anos) & $54,50 \pm 15,95$ \\
\hline $\begin{array}{l}\text { Renda (salários mínimos - SM) } \\
\text { Até } 1 \text { SM } \\
1 \text { - } 3 \text { SM } \\
3 \text { - } 5 \mathrm{SM}\end{array}$ & $\begin{array}{l}1(8,34) \\
7(58,33) \\
4(33,33)\end{array}$ \\
\hline Escolaridade (anos de estudo) & $8,41 \pm 4,98$ \\
\hline $\begin{array}{l}\text { Diagnóstico primário (tipo de neoplasia) } \\
\text { Câncer de TGl } \\
\text { Outros tipos }\end{array}$ & $\begin{array}{c}10(83,33) \\
2(16,67)\end{array}$ \\
\hline $\begin{array}{l}\text { Comorbidades } \\
\text { Hipertensão arterial } \\
\text { Obesidade } \\
\text { Desnutrição } \\
\text { Outros } \\
\text { Sem comorbidades }\end{array}$ & $\begin{array}{l}4(33,33) \\
2(16,68) \\
1(8,33) \\
1(8,33) \\
4(33,33)\end{array}$ \\
\hline $\begin{array}{l}\text { Potencial emético dos quimioterápicos } \\
\text { Moderado } \\
\text { FOLFOX } \\
\text { FOLFIRI } \\
\text { FOLFIRI+Bevacizumabe } \\
\text { 5FU + Bevacizumabe+Oxaliplatina } \\
\text { 5FU + Irinotecano+Folinato de Cálcio } \\
\text { Alto } \\
\text { Ciclofosfamida + Rituximabe+Fludarabina }\end{array}$ & $\begin{array}{c}11(91,67) \\
7(58,33) \\
1(8,33) \\
1(8,33) \\
1(8,33) \\
1(8,33) \\
1(8,33) \\
1(8,33)\end{array}$ \\
\hline $\begin{array}{l}\text { ASG-PPP } \\
\text { Estágio A } \\
\text { Estágio B } \\
\text { Estágio C }\end{array}$ & $\begin{array}{l}5(41,67) \\
6(50,00) \\
1(8,33)\end{array}$ \\
\hline IMC (kg/m²) & $23,58 \pm 5,95$ \\
\hline
\end{tabular}

ASG-PPP (Avaliação Subjetiva Global Produzida pelo Próprio Paciente); IMC (Indíce de Massa Corporal). 
Foi observado predomínio de protocolos quimioterápicos de moderado potencial emético $(91,67 \%)$, sendo mais incidente o FOLFOX (oxaliplatina/fluoracil/ácido folínico), o que torna a amostra homogênea quanto ao potencial emetogênico ${ }^{6}$. O produto utilizado na intervenção, assim como em outros estudos, não demosntrou toxicidade aos participantes, pois em momento algum houve relatos de eventos adversos ${ }^{13,8}$.

A ocorrência de náuseas e vômitos, número de episódios de vômitos e a intensidade da náusea, nos parâmetros agudos e tardios do momento basal estão apresentados nas Tabelas 2 e 3, demonstrando que não houve diferença entre os grupos ao início do estudo.

Foi encontrada diferença entre os grupos gengibre e placebo referente à intensidade da náusea aguda, apresentando maior redução desta no grupo que recebeu o placebo (Placebo: 1,25 $\pm 2,00$; Gengibre: 3,67 $\pm 3,39$, $p=0,033)$. A comparação do efeito da náusea aguda e tardia está apresentada na Figura 3.

Tabela 2. Parâmetros agudos e tardios de ocorrência de náusea e vômitos no momento inicial.

\begin{tabular}{lccc}
\hline & Placebo & Gengibre & Valor de $\boldsymbol{p}^{*}$ \\
\hline Agudo & $(\%)$ & $(\%)$ & 0,511 \\
Ocorrência de Náusea & 100 & 75 & 0,209 \\
Ocorrência de Vômitos & 0 & 50 & 1,000 \\
Tardio & & & 0,513 \\
Ocorrência de Náusea & 75 & 87,5 & 25 \\
Ocorrência de Vômitos & 0 & & \\
\hline
\end{tabular}

* Os valores mensurados foram comparados com o Teste Exato de Fischer.

Tabela 3. Parâmetros agudos e tardios de episódios de vômitos e intensidade da náusea no momento inicial.

\begin{tabular}{lccc}
\hline & Placebo & Gengibre & Valor de $\boldsymbol{p}^{*}$ \\
Agudo & Mediana (IQ) & Mediana (IQ) & \\
$\quad$ Episódios de vômitos & 0 & $0,5(0-1,5)$ & 0,128 \\
$\quad$ Intensidade da náusea & $7(5-9,25)$ & $7(3,75-8)$ & 0,604 \\
Tardio & & & \\
$\quad$ Episódios de vômitos & 0 & $0(0-0,25)$ & 0,361 \\
$\quad$ Intensidade da náusea & $5,5(3-7,75)$ & $5,5(3,5-7,25)$ & 1,000 \\
\hline
\end{tabular}

*Os valores mensurados foram comparados com Wilcoxon Signed Rank.

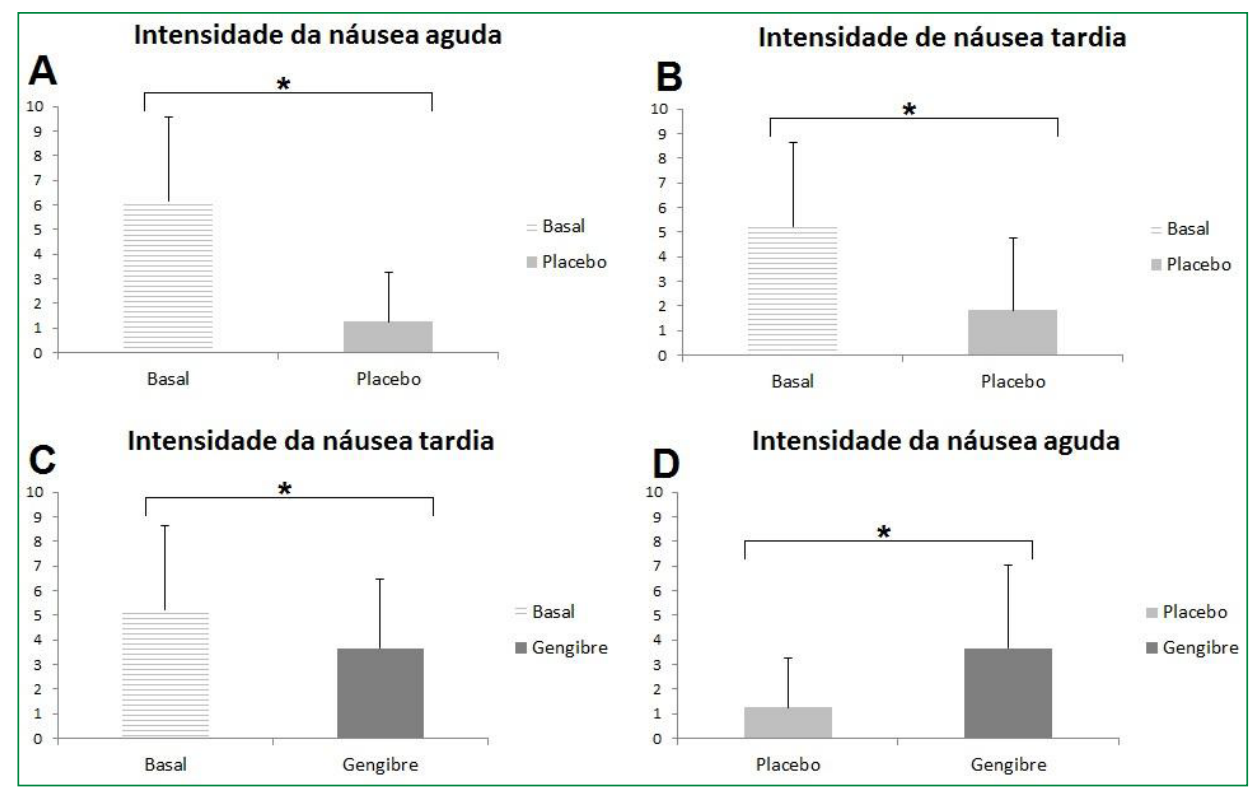

*Diferença com $p<0,05$.
Figura 3. Intensidade da náusea aguda e tardia. A: intensidade da náusea aguda entre momento inicial e placebo; $\mathrm{B}$ : intensidade da náusea tardia entre momento inicial e placebo; $\mathrm{C}$ : intensidade da náusea tardia entre momento inicial e gengibre; D: intensidade da náusea aguda entre placebo e gengibre. As análises foram realizadas com teste de Wilcoxon Signed Rank para as variáveis ordinais e o teste de McNemar foi utilizado para as variáveis qualitativas. 
Entre os grupos, não houve diferença quanto ao número de episódios de vômitos agudos $(p=0,181)$ e tardios $(p=0,586)$, ocorrência de vômitos agudos $(p=0,479)$ e tardios $(p=1,000)$, ocorrência de náusea aguda $(p=0,221)$ e tardia $(p=0,134)$. Também não houve diferença quanto à intensidade da náusea tardia $(p=0,139)$.

Houve diferença na redução na intensidade da náusea aguda no grupo que recebeu placebo quando comparado ao momento basal (Basal: 6,17 $\pm 3,41$; Placebo: 1,25 $\pm 2,00$; $p=0,014)$, assim como redução na intensidade da náusea tardia (Basal: 5,25 $\pm 3,41$; Placebo: 1,83 $\pm 2,92 ; p=0,032$ ).

Entre o placebo e o momento basal não houve diferença nos parâmetros ocorrência de vômitos agudos $(p=0,371)$ e tardios $(p=1,000)$, ocorrência de náusea aguda $(p=0,114)$ e tardia $(p=0,074)$ e episódios de vômitos agudos $(p=0,095)$ e tardia $(p=1,000)$.

Quando comparado o grupo que recebeu a suplementação de gengibre com o momento basal houve diferença quanto à intensidade de náusea tardia (Basal: 5,25 $\pm 3,41$; Gengibre: $3,66 \pm 2,81 ; p=0,017$ ).

Entre o gengibre e o momento basal não houve diferença quanto à ocorrência de vômitos agudos $(p=1,000)$ e tardios $(p=1,000)$, ocorrência de náusea aguda $(p=0,617)$ e tardia $(p=1,000)$, episódios de vômitos agudos $(p=1,000)$ e tardios $(p=1,000)$ e intensidade da náusea aguda $(p=0,118)$.

\section{DISCUSSÃO}

Neste estudo, foi identificado que o gengibre não conferiu efeito protetor quanto às náuseas e vômitos induzidos pela quimioterapia e, ao comparar os dois grupos, foi observado que a redução da intensidade de náusea aguda foi maior no grupo placebo.

O efeito placebo é uma resposta complexa que envolve alterações neurobiológicas geradas por uma variedade de fenômenos mentais, como expectativa, recompensa e redução da ansiedade, que podem ser moduladas por desejo, motivação e memória ${ }^{14}$. No presente estudo, a redução de náuseas tardias foi maior no grupo placebo, o que pode ser justificado por envolver a crença dos participantes em participar de um processo benéfico. Em um estudo recente que avaliou o efeito placebo em participantes com câncer apresentando fadiga, foram verificados efeitos positivos com o placebo $^{15}$.

Alguns estudos com pacientes em quimioterapia, utilizando doses de extrato seco padronizado de gengibre variando entre $160 \mathrm{mg} / \mathrm{dia}^{16}, 1,0$ a 2,0 g/dia ${ }^{8,13}$ e 1,2 g/dia ${ }^{10}$, por 2 ciclos de quimioterapia, 3 dias, 5 dias e 5 ciclos de quimioterapia, respectivamente, também não encontraram diferenças significativas em náuseas e vômitos, com a suplementação do gengibre.
Em um estudo com 88 pacientes com câncer foi ofertado $10 \mathrm{mg}$ de 6 -gingerol por 12 semanas $^{17}$. Como resultado, houve melhora na taxa de resposta completa em náuseas e vômitos induzidos por quimioterapia, no apetite e na qualidade de vida. A suplementação com o princípio ativo do gengibre isolado e o período de intervenção de 12 semanas pode ter associação com os resultados positivos encontrados, visto que em nosso trabalho foi utilizado gengibre em forma de extrato seco e por um período bem menor.

Além disso, as alterações nas concentrações de compostos bioativos das plantas podem ocorrer devido a alguns fatores, tais como a localização geográfica em que foi cultivado; o método de preparo do material e a forma do produto utilizado (fresco, óleo essencial ou desidratado) ${ }^{18}$. Portanto, ainda não se pode afirmar que o gengibre é eficaz sobre náuseas e vômitos em pacientes em QT, tampouco indicá-lo com essa finalidade.

Outro fator importante é que a toxicidade das terapias pode ser exacerbada pela desnutrição reduzindo assim a tolerância e a efetividade do tratamento antineoplásico. Sabe-se que pacientes que apresentam perda de peso durante a quimioterapia relatam maior toxicidade e sintomatologia do que aqueles que apresentam peso estável ${ }^{19}$. Em nossa amostra, a frequência de desnutrição grave (ASG-PPP - estágio C) foi baixa, o que pode ter influenciado na tolerância dos pacientes ao regime quimioterápico e, consequentemente na intensidade das náuseas e vômitos induzidos pela quimioterapia.

Dentre os pontos fortes do estudo podemos destacar o delineamento do estudo por ser do tipo cruzado, em que os participantes eram comparados com eles mesmos, sendo submetidos a duas intervenções em momentos distintos; o fato de o estudo ser triplo cego, pois nem os pesquisadores, nem os participantes, nem o estatístico sabiam o que cada paciente recebeu em cada momento da intervenção; e a escolha do instrumento para avaliação de náuseas e vômitos, que é um instrumento de avaliação subjetiva de náuseas e vômitos, produzido e validado para esse público, pela Multinational Association on Suportive Care in Cancer (MASCC) ${ }^{11}$. Além disso, é um instrumento pequeno, de fácil entendimento e aplicabilidade por serviços que queiram acompanhar esse sintoma em seus pacientes.

Este estudo apresentou algumas limitações, tais como a utilização do extrato seco não padronizado; o tamanho reduzido da amostra comparada a outros estudos, que ocorreu devido ao número limitado de participantes que se enquadravam nos critérios de inclusão do estudo e pelo curto período para recrutamento e intervenção. Além disso, os questionários feitos no momento basal não foram aplicados novamente antes da segunda intervenção, o que aconteceu 
porque os participantes compareciam ao hospital apenas no próximo ciclo de quimioterapia, dificultando assim o contato antecipado destes com o pesquisador.

Em conclusão, verificou-se que o gengibre e o placebo reduziram a intensidade de náuseas tardias em relação ao momento basal. Porém, ao comparar os dois grupos, foi observada redução significativa apenas na intensidade de náusea aguda, e esta ocorreu no grupo placebo. Assim, não foi encontrado, com esse desenho de estudo, um efeito protetor em relação a náuseas e vômitos agudos ou tardios. Há controvérsias na literatura quanto ao efeito da suplementação de gengibre, sendo necessários mais estudos clínicos para assegurar sua indicação no manejo de náuseas e vômitos induzidos por quimioterapia.

\section{REFERÊNCIAS}

1. Soares LC, Burille A, Antonacci MH, Santana MG, Schwartz E. A quimioterapia e seus efeitos adversos: relato de clientes oncológicos. Cogitare enferm. 2009;14(4):714-9. https://doi.org/10.5380/ ce.v14i4.16388

2. Rodrigues FSS, Polidori MM. Enfrentamento e resiliência de pacientes em tratamento quimioterápico e seus familiares. Rev Bras Cancerol. 2012;58(4):619-27.

3. Barbosa MBA; Instituto Nacional de Câncer (BR). Ações de enfermagem para o controle do câncer: uma proposta de integração ensino serviço. 3르 ed. Rio de Janeiro: INCA; 2008.

4. Molassiotis A, Saunders MP, Valle J, Wilson G, Lorigan P, Wardley A, Levine E, Cowan R, Loncaster J, Rittenberg C. A prospective observational study of chemotherapy-related náusea and vomiting in routine practice in a UK cancer center. Support Care Cancer. 2008;16(2):201-8. https://doi.org/10.1007/s00520-007-0343-7

5. Roila F, Herrstedt J, Aapro M,Gralla RJ, Einhorn LH, Ballatori E, Bria E, Clarck-Snow RA, Espersen BT, Feyer P, Grunberg SM, Hesketh PJ, Jordan K, Kris MG, Maranzano E, Molassiotis A, Morrow G, Olver I, Rapoport BL, Rittenberg C, Saito M, Tonato M, Warr D. Guideline update for MASCC and ESMO in the prevention of chemotherapyand radiotherapy-induced nausea and vomiting: results of the Perugia consensus conference. Ann. Oncol. 2010;21(5):232-43. https://doi.org/10.1093/annonc/mdq194

6. Basch E, Prestrud AA, Hesketh PJ, Kris MG, Feyer PC, Prestud AA, Temin S, Lyman GH. Antiemetics: American Society of Clinical Oncology clinical practice guideline update. J Clin Oncol. 2011;29(31):4189-98. https://doi.org/10.1200/JCO.2010.34.4614

7. Sheikhi MA, Ebadi A, Talaeizadeh A, Rahmani H. Alternative methods to treat nausea and vomiting from cancer chemotherapy. Chemother Res Pract. 2015;2015:1-6. https://doi.org/10.1155/2015/818759

8. Thamlikitkul L, Srimuninnimit V, Akewanlop C, Ithimakin S, Techawanawanna S, Korphaisarn K, Chantharasamee J, Danchaivijitr P, Soparattanapaisarn N. Efficacy of ginger for prophylaxis of chemotherapy-induced nausea and vomiting in breast cancer patients receiving adriamycin-cyclophosphamide regimen: a randomized, double-blind, placebo-controlled, crossover study. Support Care Cancer. 2017;25(2):459-64. https://doi.org/10.1007/ s00520-016-3423-8

9. Ryan JL, Heckler CE, Roscoe JA, Dakhil SR, Kirshner J, Flynn PJ, Hickok JT, Morrow GR. Ginger (Zingiberofficinale) reduces acute chemotherapy induced nausea: A URCC CCOP study of 576 patients. Support Care Cancer. 2012;20(7):1479-89. https://doi. org/10.1007/s00520-011-1236-3

10. Marx W, Mccarthy AL, Ried K, Mckavanagh D, Vitetta L, Sali A, Lohning A, Isenring E. The effect of a standardized ginger extract on chemotherapy-induced nausea-related quality of life in patients undergoing moderately or highly emetogenic chemotherapy: a double blind, randomized, placebo controlled trial. Nutrients. 2017;9(8). pii: E867. https://doi.org/10.3390/nu9080867

11. MASCC. Multinational Association on Suportive Care in Cancer [Internet]. 2017 [capturado 2017 Jan 31]. Disponível em: http:// www.mascc.org

12. Gonzalez MC, Borges LR, Silveira DH, Assunção MCF, Orlandi SP. Validação da versão em português da avaliação subjetiva global produzida pelo paciente. Rev Bras Nutr Clin. 2010;25(2):102-8.

13. Zick SM, Ruffin MT, Lee J, Normolle DP, Siden R, Alrawi S, Brenner DE. Phase II trial of encapsulated ginger as a treatment for chemotherapy-induced nausea and vomiting. Support Care Cancer. 2009;17(5):563-72. https://doi.org/10.1007/s00520-008-0528-8

14. Dias EV, Sartori CR. Compreendendo o efeito placebo. Rev Bras Ciênc Saúde. 2015;5(4).

15. Hoenemeyer TW, Kaptchuk TJ, Mehta TS, Fontaine KR. Openlabel placebo treatment for cancer-related fatigue: a randomizedcontrolled clinical trial. Sci Rep. 2018;8(1):2784. https://doi. org/10.1038/s41598-018-20993-y

16. Bossi P, Cortinovis D, Rocca MC, Roila F, Seminara P, Fabi A, Canova S, Verri E, Fatigoni S, lannace A, Macci F, Ripamonti C. Searching for evidence to support the use of ginger in the prevention of chemotherapy-induced nausea and vomiting. J Altern Complement Med. 2016;22(6):486-8. https://doi.org/10.1089/acm.2015.0315

17. Konmun J, Danwilai K, Ngamphaiboon N, Sripanidkulchai B, Sookprasert A, Subongkot S. A phase II randomized doubleblind placebo-controlled study of 6-gingerol as an anti-emetic in solid tumor patients receiving moderately to highly emetogenic chemotherapy. Med Oncol. 2017;34(4):69. https://doi.org/10.1007/ s12032-017-0931-4

18. Vieira NA, Tomiotto FN, Melo GP, Manchope MF, Lima NR, Oliveira GG, Watanabe MAE. Efeito anti-inflamatório do gengibre e possível via de sinalização. Semina Ciênc Biol Saúde. 2014;35(1):149-62. https://doi.org/10.5433/1679-0367.2014v35n1p149

19. Gomes NS, Maio R. Avaliação subjetiva global produzida pelo próprio paciente e indicadores de risco nutricional no paciente oncológico em quimioterapia. Rev Bras Cancerol. 2015;61(3): 235-42. 